\title{
CLASSIFICATION ACCURACY OF SAR IMAGES FOR VARIOUS LAND COVERS
}

\author{
V. Baby Vennila' ${ }^{1}$, R. K. Gnanamurthy ${ }^{2}$ \\ ${ }^{1}$ Research Scholar, Anna university, Tamilnadu, India \\ ${ }^{2}$ Principal, SKP Engineering College, Tamilnadu, India
}

\begin{abstract}
In recent times, the mixture of K-means clustering and Artificial Neural Network Classifier (ANN) has been often and productively useful to image categorization owing to their balancing result. Though, the amount of lessons is typically wanted to be allocated physically. This correspondence presents a disciplined unconfirmed semantic categorization technique for sky-scraping declaration protectorate descriptions. We insert tag price, which can punish a answer footed on a set of labels that come into view in it by optimization of power, to the chance meadows of dormant matters, and an iterative algorithm is thereby planned to create the quantity of lessons lastly become together to an suitable height. Evaluated with additional declared categorization procedures, our technique not only can get hold of precise semantic segmentation consequences by superior level arrangements but also can mechanically allocate the quantity of fragments. The investigational consequences on a number of prospects have established its efficiency and forcefulness.
\end{abstract}

Keywords: Label cost, K-means clustering, satellite images, Artificial Neural Network classifier (ANN).

$* * *$

\section{INTRODUCTION}

Seperating protectorate descriptions into semantically significant sections, specifically, the categorization with constancy of semantics has engaged in recreation of a significant position in latest living stages. Though, physical understanding requires abundant and luxurious human being attempt. Thus, a routine and wellorganized technique for picture semantic removal turns out to be one of the most demanding troubles in distant intelligence appliances.

In categorize to attain an well-organized satisfied removal of protectorate descriptions, a lot of grouping methods straightforwardly footed on figure characteristics have been anticipated. Though, small-stage characteristics cannot exactly correspond to the semantic of descriptions. Therefore, the association among small-stage characteristics and picture semantics turn out to be an essential problem argued newly. The most of the studies listening carefully on planning small-stage characteristics to sky-scraping stage semantics and removing the hole among them. A number of instigators quarrel that clustering, such as K-means clustering, which were initially urbanized for matter detection in manuscript field, are capable to achieve this type of effort According to the clusters, characteristics are copied as "illustration expressions" by vector quantization, and descriptions are observed as papers and copied as combination of pixel values. The categorization or segmentation consequences resulting as of them can be more dependent on satisfied consistency. In accumulation, the effectual calculation footed on estimated deduction techniques can map the skyscraping dimensional characteristic add up into smalldimensional feature values, which creates clustered images extraordinary. It has exposed that instead of descriptions color assortments outperforms utilizing small stage characteristics. It has been accounted that the explanation presentation of huge protectorate descriptions can profit from feature values.

Even though the unbeaten submission and inspiring presentation of image forms, they undergo from deprived spatial consistency for the reason that of the self-government supposition of illustration expressions and descriptions. A random selection of image file has been engaged for rectifying this trouble as the spatial sequence among adjacent sections in a picture is in that way imposed. The investigational consequences of take charge of and inadequately managed locations show that the two joint copies are balancing and the segmentation and acknowledgment correctness is clearly enhanced.

Though, the abovementioned technique was beforehand planned for managed picture categorization. In addition, the quantity of lessons, which has an important power in excess of categorization outcome, is typically specifically footed on an aforementioned or well-informed task in the majority categorization methods. Furthermore, for the reason that of the skyscraping motion, satellite descriptions have comparatively comfortable sequence. The picture semantics of big arrangement cannot be symbolized correctly by merely using satellite image models.

We expand the mixture of image models of unsubstantiated protectorate picture categorization, more exactly, utilizing an over dormant focus tags, which are attained by K-means clustering, to improve the spatial consistency sequence. In addi- 
tion, the tag rate declared which has an outstanding simplification and can give its have possession of involvement to picture segmentation, is added to the random fields of features. Mechanically segmenting a picture into consistent divisions is forever a significant subject in segmentation. The smallest amount report duration measure was first proposed for unsubstantiated segmentation, to symbolize the picture more efficiently. In the instigators have sharp out that utilizing $\alpha$ growth can be additional influential, for the reason that the segmentation footed on such a technique relies on outline development and clear integration of neighboring sections. We initiate an iterative procedure over image topics, from side to side which the quantity of lessons is ultimately suitably united. The entire procedure works routinely, as an alternative of pretentious it earlier as a stable. For the moment, for the reason that of the total combination purpose of tag rate, both the constancy of semantics is glowing set aside and the in excess of flat consequence is avoided. Our investigational consequences demonstrate that, based on the incidental sensible quantity of fragments, the proposed technique outperforms extra declared techniques. Furthermore, the competence of semantics removal is also in that way established.

\section{RELATED WORK}

LDA (Latent Dirichlet allocation) is initially urbanized for manuscript paper modeling, which is a generative probabilistic technique for groups of separate data. Contrary to the pLSA (probabilistic latent semantic analysis) method, LDA creates it probable to allocate likelihood to papers in the preparation quantity and can simply simplify to novel papers. It considers that each manuscript is in the shape of combination of latent topics and each topic is produced from expressions. All issues strained from terms of a manuscript are unspecified to be provisionally self-governing of every technique.

In LDA, every manuscript is a series of $N$ words $S n$, denoted by $S=\{S 1, S 2, \ldots, S N\}$. For the reason that of the appliance of such total generative form from manuscript field to picture domain, some terminologies require to be distinct: The corpus $D$ denotes the image data set, papers communicate to sub images, and word $w n$ is equivalent to scrap of sub images. Each picture is thereby observed as a series of $N$ visual words. Its generative process is as follows.

1) Choose a $K$-dimensional Dirichlet random variable $\theta \sim$ $\operatorname{Dir}(\alpha)$; here, $K$ denotes the number of topics.

2) For each of the $N$ words $w n$ :

a) Choose a topic $z n \sim$ Multinomial $(\theta)$.

b) Choose a word $w n$ from $P(w n \mid z n, \beta)$, a multinomial probability conditioned on the topic $z n$ with probability matrix $\beta$.

Here, $M$ is the amount of documents in corpus, and $\alpha$ and $\beta$ are hyper parameters. Unfortunately, (1) is intractable for exact parameter inference. The corresponding solution is using variational inference or some other approximate inference algorithms, such as Gibbs sampling.
The lack of spatial information is an obvious drawback of topic models due to the conditional independence assumption. Using the MRF model to capture the local correlations between all spatially adjacent neighbors is an effective way to solve this problem.

There exist various classification methods for classifying satellite imagery which includes Maximum likelihood classifier, kNN classifier, parallel piped classifier and fuzzy classifier. Multilayer perceptrons have been applied to variety of problems in image processing, including optical character recognition [7] and medical diagnosis [3, 4]. A. Tzotsos and D. Argialas, has suggested Support Vector Machine classification technique for Object Based Image Analysis (OBIA) for supervised classification of the satellite imagery using object based representation [1]. X. Gigandet et.al. have suggested region based satellite image classification that combines unsupervised segmentation with supervised segmentation using Gaussian hidden Markov random field and Support Vector Machine [8].

\section{OUR APPROACH}

In this section, we describe our approach. First, we introduce the K-means clustering model. Second, we explain ANN classifier works, which is based on pixel labels and feature vectors.

\subsection{K-Means Clustering}

This method begins with a few groups of pixels in the characteristic vectors, each of them described by its midpoint. The initial step consists in assigning each pixel to the adjacent group. In the next step the new midpoints are calculated with the novel groups. These two steps are frequent in anticipation of junction. The essential step of k-means grouping is straightforward. In the commencement decide a quantity of groups $\mathrm{K}$ and suppose the centroid or midpoint of these groups. Get any chance of articles as the original centroids or the primary $\mathrm{K}$ objects in series can also provide as the primary centroids.

The K-means clustering method will do the three steps below in anticipation of convergence.

Iterate until stable (= no object move group)

1. Decide the centroid organize

2. Decide the space of each thing to the centroids.

3. Collection the object based on minimum distance.

After accomplishment of $\mathrm{K}$-means clustering, the consequences of 'segmentation and classification' are accumulated and then identical descriptions are given to the neural network classifier for further study. 


\subsection{Artificial Neural Network (ANN) Classifier}

The ANN classifier is a simplification of the least mean square method that transforms network heaviness to reduce the mean squared error among the preferred and real productions of the network. ANN employs managed knowledge in which the network is skilled utilizing information for which contributions as well as preferred productions are recognized. On one occasion skilled, the network weights are ice-covered and can be employed to calculate production worth's for new contribution models.

\subsubsection{Steps for ANN Classifier}

Start with randomly chosen weights.

While Mean Square Error is unacceptable and computational limits are not go beyond, do for every contribution model.

1. Compute hidden node inputs $\left(\right.$ net $\left._{p, j}^{(I)}\right)$

2. Compute hidden node outputs $\left(X_{p, j}^{(I)}\right)$

3. Compute inputs to the output nodes $\left(\right.$ net $\left._{p, k}^{2}\right)$

4. Compute the network outputs $\left(O_{p, k}\right)$

5. Compute the error between $O_{p, k}$ and desired output $d_{p, k}$

6. Modify the weights between hidden and output nodes: $\Delta w_{k, j}^{(2,1)}=\eta\left(d_{p, k}-O_{p, k)} \delta^{1}\left(n e t_{p, k}^{2}\right) X_{p, j}^{(1)}\right.$

7. Modify the weights between input and hidden nodes

End for

End-while

\section{EXPERIMENTAL RESULTS}

Classification using above mentioned K-means clustering and ANN classifier:

1. Read the input satellite image and display it.

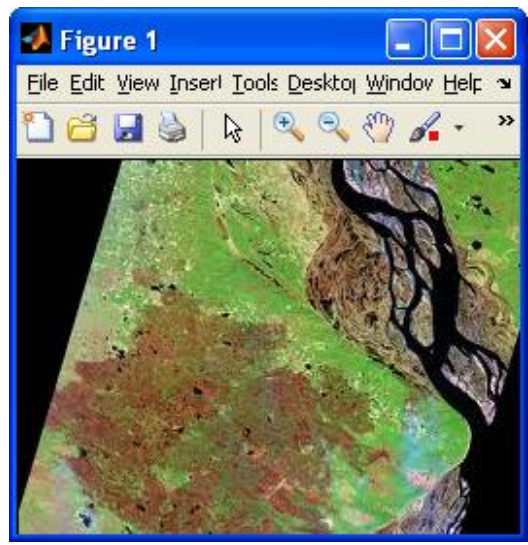

Fig.1. Input Satellite Image

2. Preprocessing of an Image
Preprocessing is the method to include smoothing, sampling, and filtering. In this method we are going to do reduce the noises by using filters. Filtering is used to remove the unwanted noises in an image.

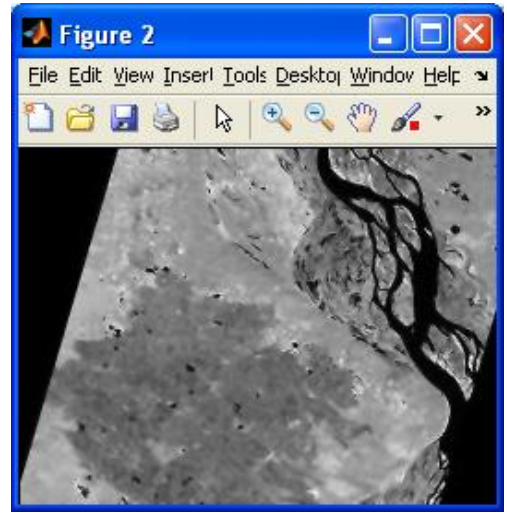

Fig.2. Preprocessing of an Image

\section{Color Separation}

First enhancing color separation of satellite image using decorrelation stretching is carried out and then the regions are grouped into a set of five classes using K-means clustering algorithm.
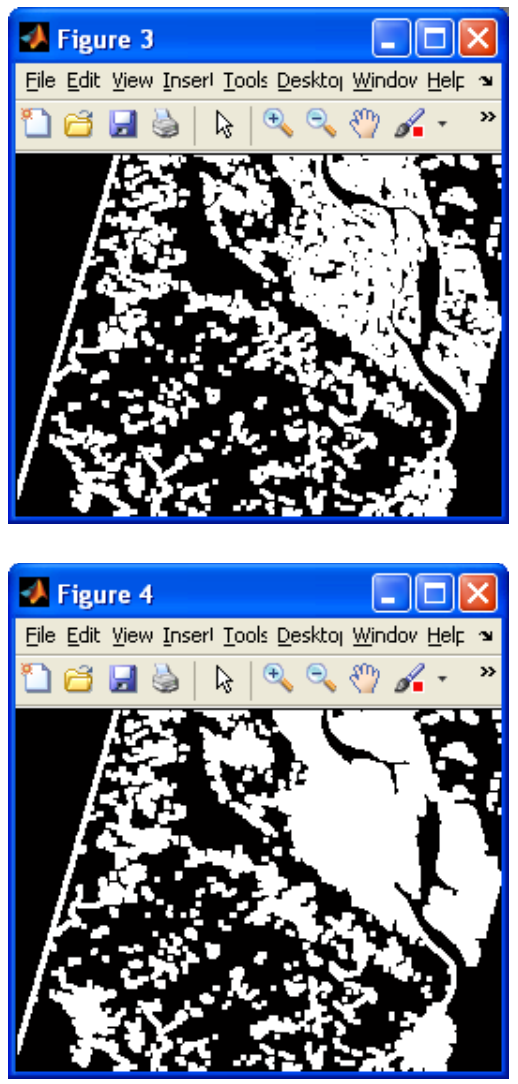

Fig.3.Color Separation 


\section{Convert Image from RGB Color Space to $L * a * b *$ Color Space}

In land cover changes in an RGB image, first converts the image to $L^{*} a^{*} b^{*}$ color space to separate intensity information into a single plane of the image, and then calculates the local range in each layer.

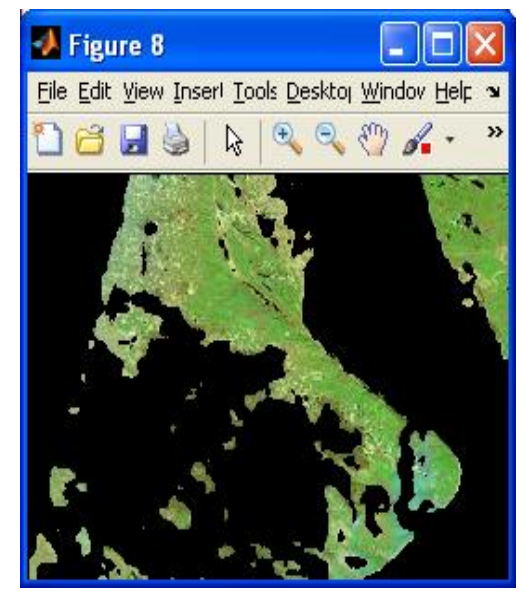

Fig.4. RGB color space to $\mathrm{L}^{*} \mathrm{a} * \mathrm{~b}$ Color space

\section{Classify the Colors in ' $a * b * '$ Space Using ANN}

Artificial Neural network classifier requires that you specify the number of clusters to be partitioned and a distance metric to quantify how close two objects are to each other. Since the color information exists in the 'a*b' space, your objects are pixels with 'a*' and 'b*' values.

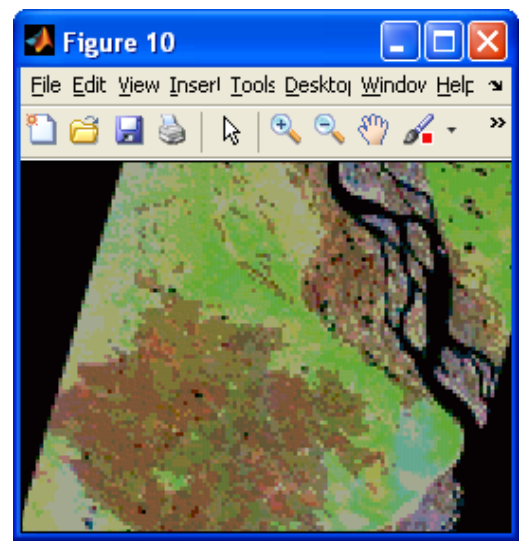

Fig.5.Classify the colors using ANN

\section{Find the membership function for satellite images}

Neural Network classification techniques allow pixels to have membership in more than one class and therefore better represent the imprecise nature of the data. This technique produces a substantial increase in classification accuracy of urban land cover maps compared to the traditional maximum likelihood classification approach.

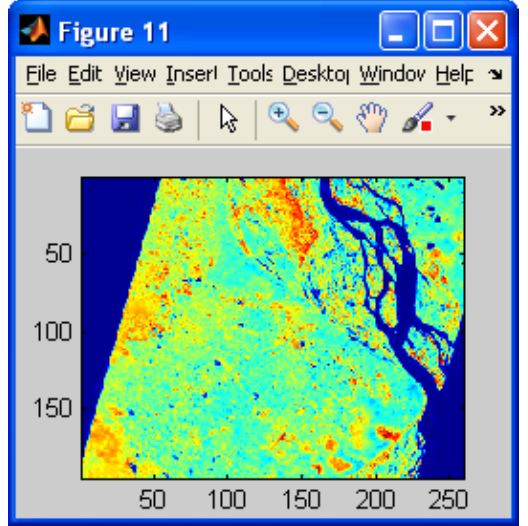

Fig.6. Membership Function for Satellite Images

\section{CONCLUSIONS}

K-means clustering and ANN classifier are considered in feature and realized on a variety of record for the segmentation and categorization principle. It is establish that $\mathrm{K}$ - means clustering provides extremely sky-scraping precision, but it is helpful for distinct record at a occasion. Whereas neural network is helpful for numerous records, once it is skilled for it. Neural network also offers superior precision.

\section{REFERENCES}

[1]. Flayed Sabine and W.H.Freeman, "Remote sensing, Principles and Interpretation".

[2]. S.N. Sivanandam \& Sumathi \& S. N. Deepa, "Introduction to Neural Networks using Matlab 6.0".

[3]. Bernd Jahne and Horst HauBecker (2007), Computer Vision and Applications, Academic Press.

[4]. Dhawan, A. P., Chitre, Y., Kaiser, C., and Moskowitz, M., (1996). Analysis of mammographic micro calcifications using gray-level image structure features, IEEE Trans. Medical Imaging, 246-259.

[5]. Rafael C. Gonzalez \& Richard E.Woods, "Digital Image Processing".

[6]. Rui Xu and Donald Wunsch, "Survey of Clustering Algorithms", IEEE, Vol. 16, No.3, pp.645-678, May 2005.

[7]. Säckinger, E., Boser, B. E., Bromley, J., LeCun, Y., and Jackel, L. D., (1992), Application of an ANNA neural network chip to high-speed character recognition, IEEE Trans. Neural Networks, 498-505.

[8]. X. Gigandet et. al., Region-Based Satellite Image Classification: Method and Validation, Proc. of IEEE International Conference on Image Processing, 2005, 832-835. 